\title{
Bowel Necrosis in the Setting of COVID-19
}

\section{Rajshri M. Gartland ${ }^{1} \cdot$ George C. Velmahos ${ }^{1}$}

Received: 23 April 2020 / Accepted: 23 April 2020 / Published online: 3 May 2020

(C) 2020 The Society for Surgery of the Alimentary Tract

As the Coronavirus Disease 2019 (COVID-19) pandemic unfolds, extra-pulmonary manifestations of the disease in critically ill, intubated patients have been observed, including hypercoagulability, acute renal failure, and myocardial injury. ${ }^{1-3}$ This is a case of a COVID-19-positive patient who presented with catastrophic bowel necrosis in the setting of patent mesenteric vessels.

The patient was a 47-year-old man with past medical history notable for type 2 diabetes mellitus who presented to the emergency department with 5 days of shortness of breath, cough, and fevers. He was intubated on arrival given hypoxia and respiratory distress. He was found to test positive for COVID-19 and treated with antibiotics for methicillin-sensitive Staphylococcus aureus pneumonia. His respiratory status was steadily improving; however, 2 weeks into his intensive care unit (ICU) course, he was noted to have rising leukocytosis, hypotension requiring escalating vasopressor support, and increased abdominal distension with grimacing on palpation of his right lower quadrant. A CT scan was obtained which demonstrated evidence of small bowel ischemia with perforation (Fig. 1). His mesenteric vessels were noted to be widely patent. On CT scan, he was also incidentally noted to have a new right posterior basal segmental pulmonary embolism.
1 Department of Surgery, Massachusetts General Hospital, Harvard Medical School, Boston, MA, USA
The patient was taken urgently to the operating room for exploration. Upon entry into the abdomen, the patient was found to have necrotic bowel extending from the ligament of Treitz to the transverse colon with a perforation of the terminal ileum (Fig. 2). The necrosis had unique characteristics, including a bright yellow discoloration (rather than black or purple ischemic changes), distribution that varied between circumferential involvement and patchy involvement along the antimesenteric side of the small bowel, and clear demarcation of the borders of ischemia without anatomic transition zones (Fig. 3). The yellow necrotic areas were found to be extremely thin and friable. Given the extent of involvement and the patient's critically ill status, the injury was deemed to be unsurvivable. The abdomen was closed and the patient passed away in the ICU shortly after transitioning to comfort measures only. The family declined an autopsy.

Further pathophysiologic study of similar cases of bowel necrosis in intubated COVID-19 patients is ongoing at our institution. Given the patent mesenteric vasculature and antimesenteric pattern of necrosis, it is hypothesized that this phenomenon may occur as a result of microvascular thrombosis and associated inflammation in these patients with known hypercoagulability. The potential for direct tissue injury from

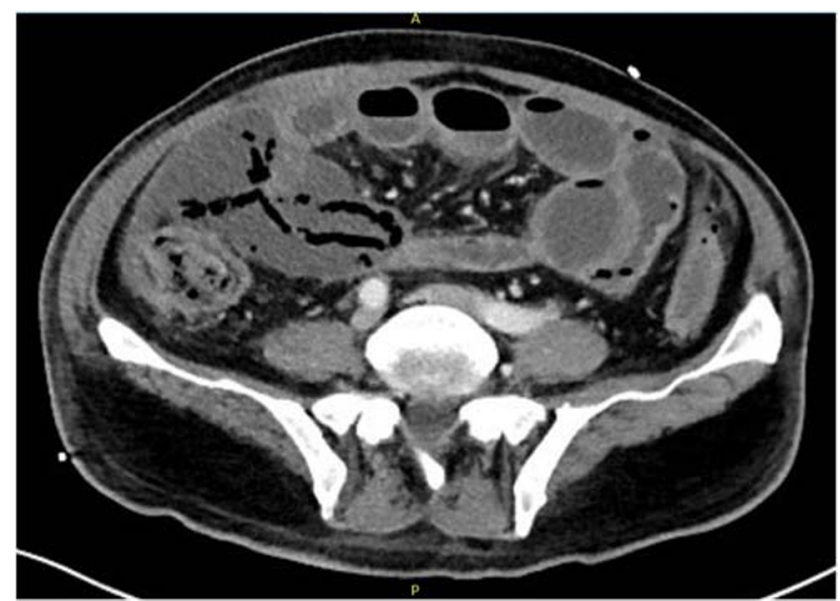

Fig. 1 CT scan demonstrating evidence of small bowel ischemia with perforation 


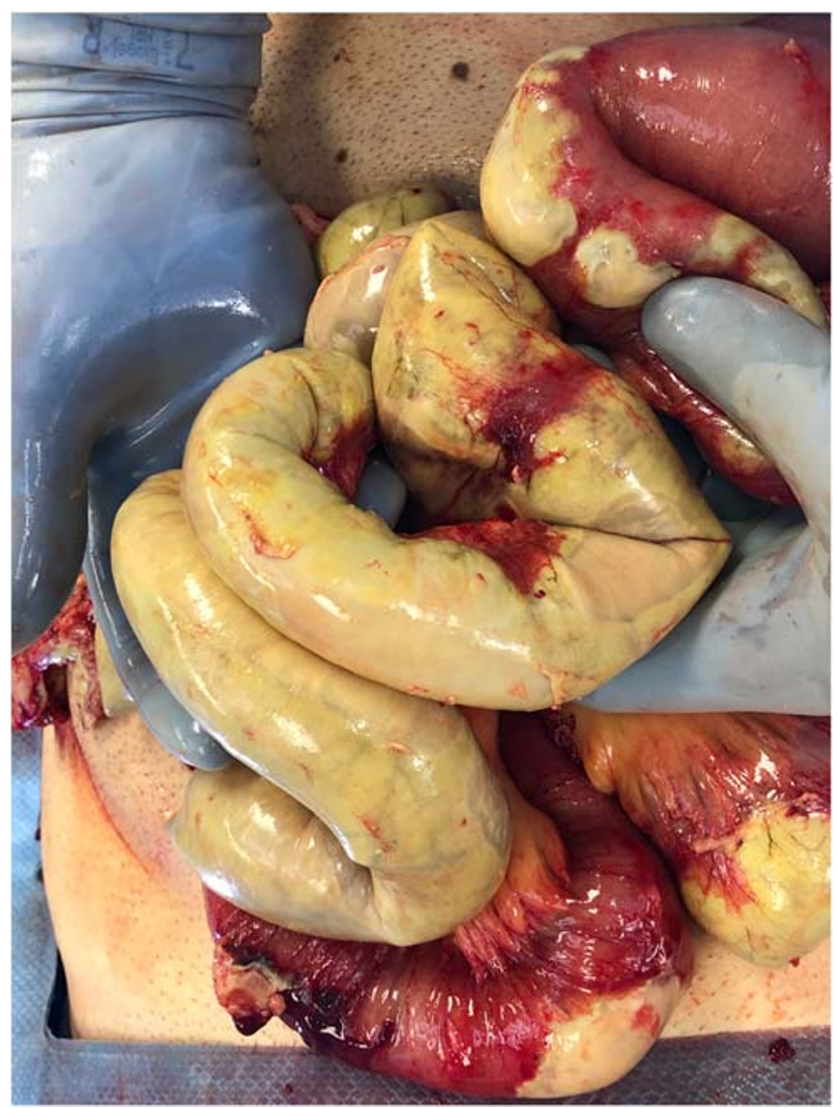

Fig. 2 Extensive bowel necrosis extending from the ligament of Treitz to the transverse colon

SARS-CoV-2 RNA in the gastrointestinal tract has not been extensively studied and may also provide insight as to the underlying mechanism of ischemia.

\section{Compliance with Ethical Standards}

Conflict of Interest The authors declare that they have no conflict of interest.

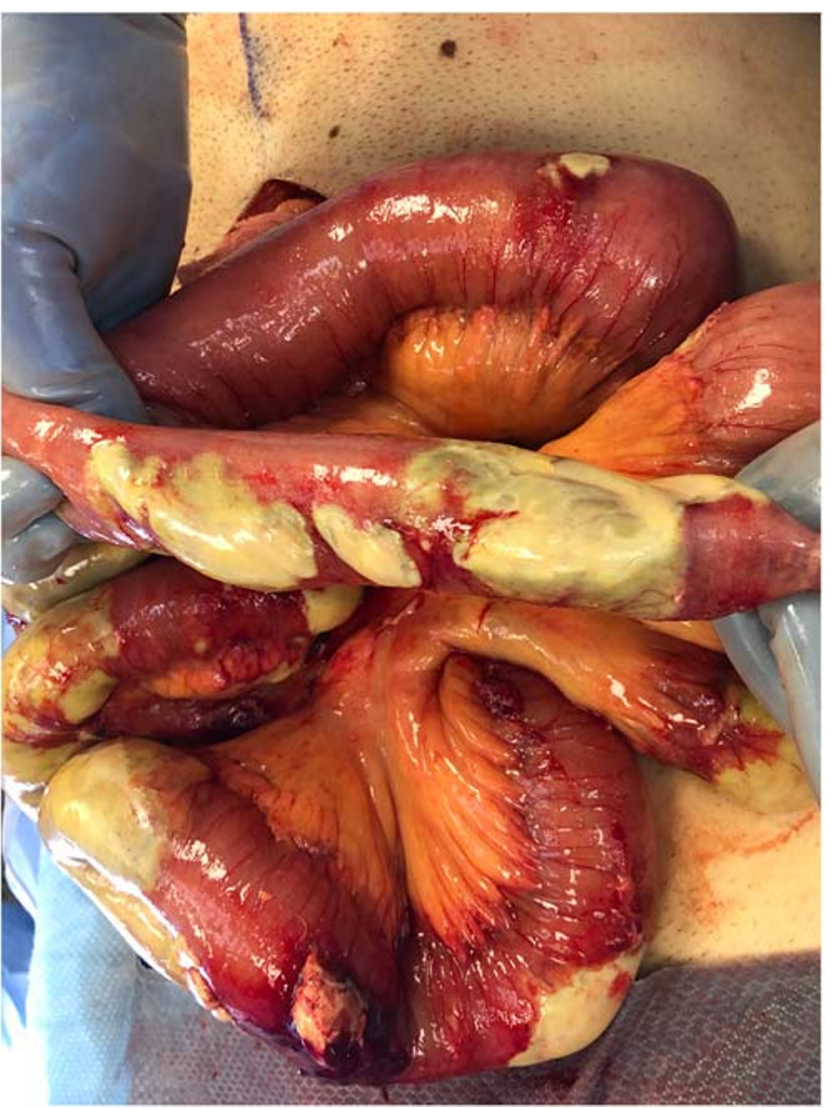

Fig. 3 Pattern of necrosis with bright yellow discoloration, clear demarcation, and distribution varied between circumferential involvement and patchy antimesenteric involvement

\section{References}

1. Spiezia L, Boscolo A, Poletto F. Covid-19-Related Severe Hypercoagulability in Patients Admitted to the Intensive Care Unit for Acute Respiratory Failure. Thromb Haemost 2020.

2. Bangalore S, Sharma A, Slotwiner A, et al. ST-Segment Elevation in Patients with Covid-19 - A Case Series. NEJM 2020.

3. Fanelli V, Fiorentino M, Cantaluppi V. Acute kidney injury in SARSCoV-2 infected patients. Crit Care 2020; 24(1):155.

Publisher's Note Springer Nature remains neutral with regard to jurisdictional claims in published maps and institutional affiliations. 